\section{Abstract PS2-22 \\ Impact of Co-payments on Patient Compliance and Persistence for Smoking Cessation Pharmacotherapy}

Scott Robinson, MA, MPH, Lovelace Clinic Foundation; Eva Lydick, PhD, Lovelace Clinic Foundation; Diana Brixner, RPh, PhD, Department of Pharmacotherapy, University of Utah; Carmen Kirkness, MPT, Department of Pharmacotherapy, University of Utah

Background/Aims: Increasing patient co-payments commonly cause decreased medication adherence. This analysis evaluated the impact of co-payment on patient compliance and persistence with smoking cessation pharmacotherapy. Methods: Patients were identified from the Lovelace Health Plan (LHP) from January 1, 2000, to June 30, 2005, by a bupropion prescription claim $(<300 \mathrm{mg} /$ day $)$, exclusive of a diagnosis for a mood disorder (ICD-9 311.X), with no more than 9 refills and with continuous enrollment 3 months prior and 12 months post the initial fill date. Patients were grouped into three co-payment groups: $<\$ 10,>\$ 10$ to $<\$ 20$ and $>\$ 20$. Compliance was calculated as the days of available therapy over indicated therapy (49 days) and persistence as the percentage of patients remaining on therapy at 15, 49 and 60 days. Patients were stratified by age, gender, ethnicity and comorbidities. Resource use and costs were collected for each group. Results: The study population consisted of 1732 subjects with 496, 330 and 292 in each of the co-payment categories and a cohort of 613 patients for whom the co-pay was not available. Patients who paid $<\$ 10$ were the least compliant, with a medication possession ratio (MPR) of 0.7325 (CI, 0.7164-0.7486), and those who paid $>\$ 20$ were the most compliant, with an MPR of 0.7646 (CI, 0.7614-0.8084). Compliance was associated with gender (0.7654 female vs. 0.7419 male), age (0.7634 age $40-64$ vs. 0.7370 age 18-39), and ethnicity (0.7650 non-Hispanic vs. 0.7220 Hispanic). Persistence at 15 days was almost $100 \%$ for all three tiers. Persistence at 49 days was 0.3919 (CI, 0.3559- 0.4424) for the lowest co-pay cohort and 0.5358 (CI, $0.4784-0.5933, P<0.001)$ for the highest co-pay cohort. Persistence at 60 days followed the same trends as at 49 days. Females aged 40-64 and non-Hispanics demonstrated greater persistency. The impact of age, gender, ethnicity, and comorbidities on the results will be presented along with the resource use and cost distributions between groups. Conclusions: The more common relationship of increased cost-sharing to decreased adherence seen in other disease states may be the inverse for smoking cessation pharmacotherapy. Patient behavioral components, such as motivation to quit, may play a larger role.

\section{Abstract PS2-23}

The Associations of Medication Adherence with Employment and Work Disability Among Patients with Relapsing Remitting Multiple Sclerosis

Kaan Tunceli, PhD, Center for Health Services Research, Henry Ford Hospital; Mirela Cerghet, MD, Henry Ford Hospital; Elizabeth A. Dobie, MPH, Henry Ford Hospital; Stanton Elias, MD, Henry Ford Hospital; Manel Pladevall, PhD, Henry Ford Hospital; Lonni Schultz, PhD, Henry Ford Hospital; Jennifer E. Lafata, PhD, Henry Ford Hospital

Background: To understand fully the economic impact of interventions designed to manage relapsing-remitting multiple sclerosis (RRMS), the impact of medication adherence on patient outcomes needs to be understood. This study examines the associations between adherence to commonly used disease modifying agents (DMAs) and employment or work disability among patients with RRMS. Methods: We used automated medical and pharmaceutical claims data along with data from patient charts to identify eligible study participants with RRMS and their adherence to DMAs. A mixed mailed/telephone survey administered to a cohort of 224 HMO insured patients with RRMS was used to solicit information on employment status and socio-demographic characteristics. A continuous measure of medication availability (CMA) expressed as the ratio of the cumulative days supply of DMA dispensed over 365 days was constructed for the year preceding survey administration. CMA $\geq 80 \%$ was used to identify patients who were adherent. We used multivariable logistic regressions to examine the associations between adherence and the probability of employment or work disability. Models controlled for patient age, gender, race, and comorbidities. Results: One hundred seventy patients responded to the survey (response rate: 76\%). Our final sample was comprised of 111 patients who had been dispensed a DMA in the year preceding survey administration. Among these, 57\% reported working at the time of the survey and $21 \%$ reported they were work disabled (i.e., had an impairment or a health problem that kept them from working). In the multivariable logistic model predicting employment status, being therapy adherent was associated with a greater likelihood of working $(\mathrm{OR}, 3.18 ; P<0.05)$. In the multivariable model predicting work disability, being therapy adherent was associated with a decreased likelihood of work disability (OR, $0.37 ; P<0.10)$. Conclusions: Our findings suggest that adherence to DMAs among RRMS patients is associated with a greater probability of employment and a lesser probability of work disability. In the assessment of the cost-effectiveness of interventions to improve medication adherence, the effects on employment and work disability of medication adherence should be considered along with those of medical care costs and other outcomes.

\section{Abstract PS2-24}

Diminishing Incentives: Drug Co-Payment Effects Over Four Years

Huihui Wang, Division of Research, Kaiser Permanente; Jie Huang, PhD, Division of Research, Kaiser Permanente; Vicki Fung, PhD, Division of Research, Kaiser Permanente; John Hsu, MD, MBA, MSCE, Kaiser Permanente Northern California

Background: There is limited information about how prescription drug cost-sharing effects change over time. Aim: In a natural experiment, we examined annual changes in prescription drug expenditures associated with having a two-tier prescription drug co-payment over a 5-year period. Methods: We examined 8224 adult Kaiser Permanente-Northern California commercially insured members with diabetes in January 2001 who were continuously enrolled from 2001-2005. In 2001, subjects paid $\$ 5$ for prescription drugs. Starting in 2002, 66\% of subjects paid $\$ 15$ for brand-name drugs and $\$ 5$ for generics (two-tier), while $34 \%$ of subjects had no changes (one-tier). There were no further changes in co-payments from 2002-2005. We used linear regression with a generalized estimation equations approach to examine annual prescription drug costs, truncated at the 99th percentile. We adjusted for patients' characteristics (age, gender, membership tenure, race/ethnicity, neighborhood social economic status and comorbidity) and secular trends (yearly indicators). We examined group differences (twotier vs. one-tier) over time using group-year interactions and estimated adjusted prescription drug costs based on the model coefficients and mean values of the covariates. Results: The mean age of subjects was 51 years; $47 \%$ were female, and $40 \%$ were white. In the baseline year, 2001, the mean number of prescription fills was 26.5: 27.0 in the two-tier group and 25.6 in the one-tier group. The adjusted annual total prescription drug costs were $\$ 1342$ and $\$ 1435$ in the two-tier and one-tier groups, respectively (difference $=-\$ 93,95 \% \mathrm{CI}$ : $-\$ 168$ to $-\$ 18$ ). In 2002 , the first year with a two-tier drug co-payment, annual drug costs decreased significantly among the two-tier group compared with the one-tier group (difference-in-difference $=-\$ 52 ; 95 \% \mathrm{CI}$ : $-\$ 97$ to $-\$ 8$ ). In subsequent years, the magnitude of the changes were small and not statistically significant relative to the baseline year (difference-in-difference=-\$38 for year two of the two-tier co-payment, $95 \% \mathrm{CI}$ : $-\$ 85$ to $\$ 10$; $-\$ 38$ for year three, $95 \% \mathrm{CI}$ : $-\$ 87$ to $\$ 11$; and $-\$ 34$, 95\%CI: $-\$ 88$ to $\$ 20)$. Conclusions: Introduction of a two-tier co-payment structure resulted in decreases in total prescription drug costs in the first year as expected. In subsequent years, however, changes in annual costs relative to baseline levels were smaller in magnitude and not statistically significant.

\section{Abstract PS2-28 \\ Using Natural Language Processing to Explore Schemes for Assessing Disease Risk from Free-Text Radiology Reports}

David S. Carrell, PhD, Group Health Center for Health Studies; Diana L. Miglioretti, PhD, Group Health Center for Health Studies; Rebecca Smith-Bindman, MD, University of California San Francisco

Background: Radiology reports are a form of clinical text that contains rich and useful information that is difficult to extract by automated means. The Cancer Text Information Extraction System (caTIES) is open-source software that uses natural language processing (NLP) techniques to identify concepts from standardized medical thesauri found in clinical text. Information derived from clinical text via NLP may be able to assist clinicians assess clinical risk of disease. We are exploring automated concept-coding of radiological exams of the abdomen in an effort to develop schemes for ordinal classification of clinical risk of diseases such as ovarian cancer. 